\title{
Extension of Busch's theorem to particle beams
}

\author{
L. Groening ${ }^{*}$ and C. Xiao \\ GSI Helmholtzzentrum für Schwerionenforschung GmbH, Darmstadt D-64291, Germany \\ M. Chung ${ }^{\dagger}$ \\ Ulsan National Institute of Science and Technology, Ulsan 44919, Republic of Korea
}

(Received 24 July 2017; published 10 January 2018)

\begin{abstract}
In 1926, H. Busch formulated a theorem for one single charged particle moving along a region with a longitudinal magnetic field [H. Busch, Berechnung der Bahn von Kathodenstrahlen in axial symmetrischen electromagnetischen Felde, Z. Phys. 81, 974 (1926)]. The theorem relates particle angular momentum to the amount of field lines being enclosed by the particle cyclotron motion. This paper extends the theorem to many particles forming a beam without cylindrical symmetry. A quantity being preserved is derived, which represents the sum of difference of eigenemittances, magnetic flux through the beam area, and beam rmsvorticity multiplied by the magnetic flux. Tracking simulations and analytical calculations using the generalized Courant-Snyder formalism confirm the validity of the extended theorem. The new theorem has been applied for fast modeling of experiments with electron and ion beams on transverse emittance repartitioning conducted at FERMILAB and at GSI. Thus far, developments of beam emittance manipulations with electron or ion beams have been conducted quite decoupled from each other. The extended theorem represents a common node providing a short connection between both.
\end{abstract}

DOI: 10.1103/PhysRevAccelBeams.21.014201

\section{INTRODUCTION}

In 1926, H. Busch applied the preservation of angular momentum for systems with cylindrical symmetry to a charged particle moving inside a region with magnetic field $\vec{B}$ [1-3]. Using conjugated momenta, the magnetic field strength is intrinsically included into the equations of motion. In linear systems, the normalized conjugated momenta $p_{x}$ and $p_{y}$ are related to the derivatives of the particle position coordinates $(x, y)$ with respect to the main longitudinal direction of motion $\vec{s}$ through

$$
\begin{aligned}
& p_{x}:=x^{\prime}+\frac{\mathcal{A}_{x}}{(B \rho)}=x^{\prime}-\frac{y B_{s}}{2(B \rho)}, \\
& p_{y}:=y^{\prime}+\frac{\mathcal{A}_{y}}{(B \rho)}=y^{\prime}+\frac{x B_{s}}{2(B \rho)},
\end{aligned}
$$

where $\overrightarrow{\mathcal{A}}$ is the magnetic vector potential with $\vec{B}=\vec{\nabla} \times \overrightarrow{\mathcal{A}}$, $B_{s}$ is the longitudinal component of the magnetic field, and $(B \rho)$ is the particle rigidity, i.e., its momentum per charge

\footnotetext{
*la.groening@gsi.de

mchung@unist.ac.kr
}

Published by the American Physical Society under the terms of the Creative Commons Attribution 4.0 International license. Further distribution of this work must maintain attribution to the author(s) and the published article's title, journal citation, and DOI. $p /(q e)$, with $p$ as total momentum, $q$ as charge number, and $e$ as elementary charge.

Busch's theorem [1-3] states that the canonical angular momentum $\tilde{l}=x p_{y}-y p_{x}$ is a constant of motion that is written in cylindrical coordinates as

$$
m \gamma r^{2} \dot{\theta}+\frac{e q}{2 \pi} \psi=\text { const }
$$

where $\gamma$ is the relativistic factor, $r$ is the radius of transverse cyclotron motion around the beam axis, $\dot{\theta}$ is the corresponding angular velocity, and $\psi$ is the magnetic flux enclosed by this motion. Busch's theorem for axially symmetric systems determines an invariant of motion of a single particle.

A general formulation of Eq. (3) has been derived in [4], which is regarded as the generalized Busch theorem

$$
\oint_{\mathcal{C}} \vec{v} \cdot d \vec{C}+\frac{e q}{m \gamma} \psi=\text { const }
$$

i.e., the path integral of the stream of possible particle velocities $\vec{v}$ along a closed contour $\mathcal{C}$ confining a fixed set of possible particle trajectories, plus the magnetic flux through the area enclosed by $\mathcal{C}$ is an invariant of the motion. Figure 1 illustrates the first part of the left-hand side of Eq. (4). Busch's theorem of Eq. (3) is the special case of this generalized form for $\mathcal{C}$ being a circle of radius $r$. It is emphasized that Eq. (4) is only valid for laminar motion.

The subsequent section derives an invariant of motion for a nonlaminar beam comprising many particles with an 


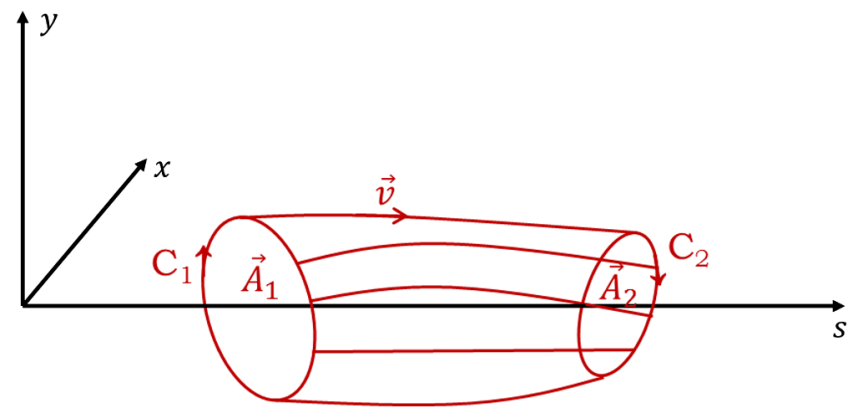

FIG. 1. The contour $\mathcal{C}$ encloses possible streams of particle velocities and encloses the area $\vec{A}$.

intrinsic transverse velocity spread, in which there is no cylindrical symmetry. The motion can pass linear coupling optical elements. An invariant is expressed through a sum of three beam properties by reformulating the invariance of the two eigenemittances introduced in 1992 by Dragt [5]. The latter holds strictly for the paraxial approximation and for mono-energetic beams as pointed out in [6], and the according assumptions are made in the following. The third section introduces the beam vorticity as one of the summands forming the invariant. In the following sections beam tracking simulations are presented as well as the application of the generalized Courant-Snyder formalism to illustrate the invariance and its constituents using a probe beam line. The paper closes in applying the extended Busch theorem for quick and precise modeling of experiments with electrons and ions on repartitioning of emittance between the two transverse degrees of freedom (planes). Throughout the paper quantities written as $\tilde{Q}$ are calculated from conjugated coordinates $\left(x, p_{x}, y, p_{y}\right)$ and those written as $Q$ are calculated from laboratory coordinates $\left(x, x^{\prime}, y, y^{\prime}\right)$; hence, $Q$ is obtained from $\tilde{Q}$ by substituting $\left(p_{x}, p_{y}\right) \rightarrow\left(x^{\prime}, y^{\prime}\right)$ in the expression defining $\tilde{Q}$.

\section{PRESERVATION OF EIGENEMITTANCES}

The two eigenemittances $\tilde{\varepsilon}_{1 / 2}$ are equal to the two projected transverse beam rms-emittances $\tilde{\varepsilon}_{x / y}$, if and only if there are no correlations between the two transverse planes. Eigenemittances can be obtained by solving the complex equation

$$
\operatorname{det}\left(J \tilde{C}-i \tilde{\varepsilon}_{1 / 2} I\right)=0
$$

where $I$ is the identity matrix and

$$
\tilde{C}=\left[\begin{array}{cccc}
\left\langle x^{2}\right\rangle & \left\langle x p_{x}\right\rangle & \langle x y\rangle & \left\langle x p_{y}\right\rangle \\
\left\langle x p_{x}\right\rangle & \left\langle p_{x}^{2}\right\rangle & \left\langle y p_{x}\right\rangle & \left\langle p_{x} p_{y}\right\rangle \\
\langle x y\rangle & \left\langle y p_{x}\right\rangle & \left\langle y^{2}\right\rangle & \left\langle y p_{y}\right\rangle \\
\left\langle x p_{y}\right\rangle & \left\langle p_{x} p_{y}\right\rangle & \left\langle y p_{y}\right\rangle & \left\langle p_{y}^{2}\right\rangle
\end{array}\right],
$$

$$
J=\left[\begin{array}{cccc}
0 & 1 & 0 & 0 \\
-1 & 0 & 0 & 0 \\
0 & 0 & 0 & 1 \\
0 & 0 & -1 & 0
\end{array}\right]
$$

Second moments $\langle u v\rangle$ are defined through a normalized distribution function $f_{b}$ as

$$
\langle u v\rangle=\iiint \int f_{b}\left(x, p_{x}, y, p_{y}\right) \cdot u v \cdot d x d p_{x} d y d p_{y}
$$

and projected rms-emittances by [7]

$$
\tilde{\varepsilon}_{u}^{2}:=\left\langle u^{2}\right\rangle\left\langle p_{u}^{2}\right\rangle-\left\langle u p_{u}\right\rangle^{2} .
$$

For two degrees of freedom, the two eigenemittances can be calculated from [8]

$\tilde{\varepsilon}_{1 / 2}=\frac{1}{2} \sqrt{-\operatorname{tr}\left[(\tilde{C} J)^{2}\right] \pm \sqrt{\operatorname{tr}^{2}\left[(\tilde{C} J)^{2}\right]-16 \operatorname{det}(\tilde{C})}}$.

As the two eigenemittances are preserved for the symplectic transformation [5], the sum of their squares is preserved as well, i.e.,

$$
\begin{aligned}
\tilde{\varepsilon}_{1}^{2}+\tilde{\varepsilon}_{2}^{2} & =-\frac{1}{2} \operatorname{tr}\left[(\tilde{C} J)^{2}\right] \\
& =\tilde{\varepsilon}_{x}^{2}+\tilde{\varepsilon}_{y}^{2}+2\left(\langle x y\rangle\left\langle p_{x} p_{y}\right\rangle-\left\langle y p_{x}\right\rangle\left\langle x p_{y}\right\rangle\right) \\
& =\text { const. }
\end{aligned}
$$

The definitions of $p_{x}$ and $p_{y}$ are plugged into Eq. (6) and Eq. (10) is used afterwards. Expanding Eq. (11) and using

$$
\varepsilon_{1}^{2}+\varepsilon_{2}^{2}=\left(\varepsilon_{1}-\varepsilon_{2}\right)^{2}+2 \cdot \varepsilon_{4 d},
$$

together with the invariance of the four-dimensional rms-emittance $\varepsilon_{4 d}^{2}=\operatorname{det}(C)=\left(\varepsilon_{1} \varepsilon_{2}\right)^{2}$, finally leads to

$$
\begin{aligned}
& \left(\varepsilon_{1}-\varepsilon_{2}\right)^{2}+\left[\frac{A B_{s}}{(B \rho)}\right]^{2} \\
& \quad+2 \frac{B_{s}}{(B \rho)}\left[\left\langle y^{2}\right\rangle\left\langle x y^{\prime}\right\rangle-\left\langle x^{2}\right\rangle\left\langle y x^{\prime}\right\rangle+\langle x y\rangle\left(\left\langle x x^{\prime}\right\rangle-\left\langle y y^{\prime}\right\rangle\right)\right] \\
& =\text { const, }
\end{aligned}
$$

where $A:=\sqrt{\left\langle x^{2}\right\rangle\left\langle y^{2}\right\rangle-\langle x y\rangle^{2}}$ is the rms-area of the beam divided by $\pi$. In the following, only laboratory coordinates are used, as the extended theorem will be applied to experiments that used these coordinates.

Equation (13) shows that changing both transverse eigenemittances can be achieved through longitudinal magnetic fields as was proposed first in [9], where the 


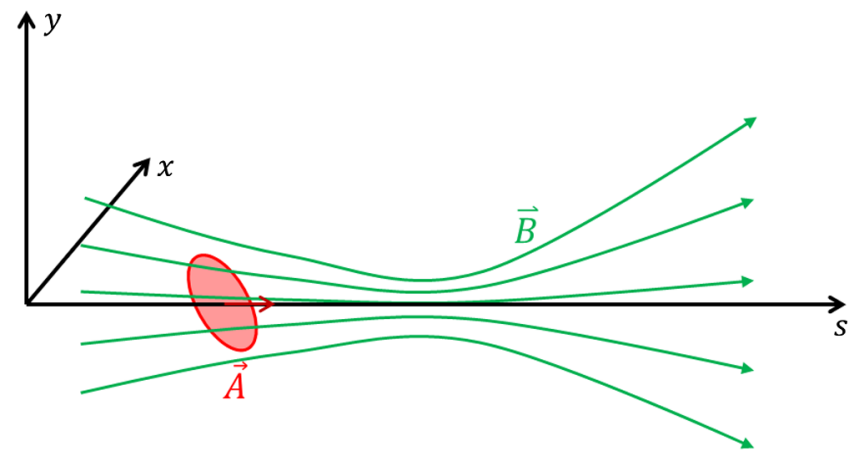

FIG. 2. Magnetic flux through the beam rms-area $\vec{A}$. Transverse field components do not contribute to the flux as they are perpendicular to the normal of the area.

beam is created inside a region of longitudinal field being emerged afterwards into a region without a field. Successful experimental demonstration of this concept was reported in [10]. The method has been applied to create very flat electron beams with aspect ratios of up to 100 [11]. Its advanced analytical description revealed that beams can be modeled in an analogue way to beams of light in planar or circular modes [12] with possible applications in circular colliders [13]. It was also proposed for ions being emerged into the solenoid field of an electron-cyclotron-resonance source to create beams of very low horizontal emittances that will allow for high-resolution spectrometers [14]. By placing a charge state stripper, i.e., changing $(B \rho)$ inside a solenoid, transverse emittance was adjustably transferred from one plane into the other one [8,15-17].

The first term of the left-hand side of Eq. (13) is the squared difference of the beam eigenemittances in the laboratory coordinates. The second term is basically the square of the magnetic flux through the beam rmsarea $\vec{A}$ as illustrated in Fig. 2. In the following, it is shown that the essential part of the third term

$$
\mathcal{W}_{A}:=\left\langle y^{2}\right\rangle\left\langle x y^{\prime}\right\rangle-\left\langle x^{2}\right\rangle\left\langle y x^{\prime}\right\rangle+\langle x y\rangle\left(\left\langle x x^{\prime}\right\rangle-\left\langle y y^{\prime}\right\rangle\right)
$$

is the rms-averaged beam vorticity multiplied by the twofold beam rms-area.

\section{BEAM VORTICITY}

We choose the ansatz assigning $\mathcal{W}_{A}$ to the rotation $(\vec{\nabla} \times)$ of the mean, i.e., averaged over $\left(x^{\prime}, y^{\prime}\right)$ space, beam angle $\overrightarrow{r^{\prime}}(x, y, s)$ being integrated over the beam rms-area, and finally multiplied by the twofold beam rms-area:

$$
\mathcal{W}_{A}=2 A \int_{A}\left[\vec{\nabla} \times \overrightarrow{r^{\prime}}(x, y, s)\right] \cdot d \vec{A}
$$

being equivalent to

$$
\mathcal{W}_{A}=2 A \oint_{\mathcal{C}} \overrightarrow{r^{\prime}}(x, y, s) \cdot d \vec{C}
$$

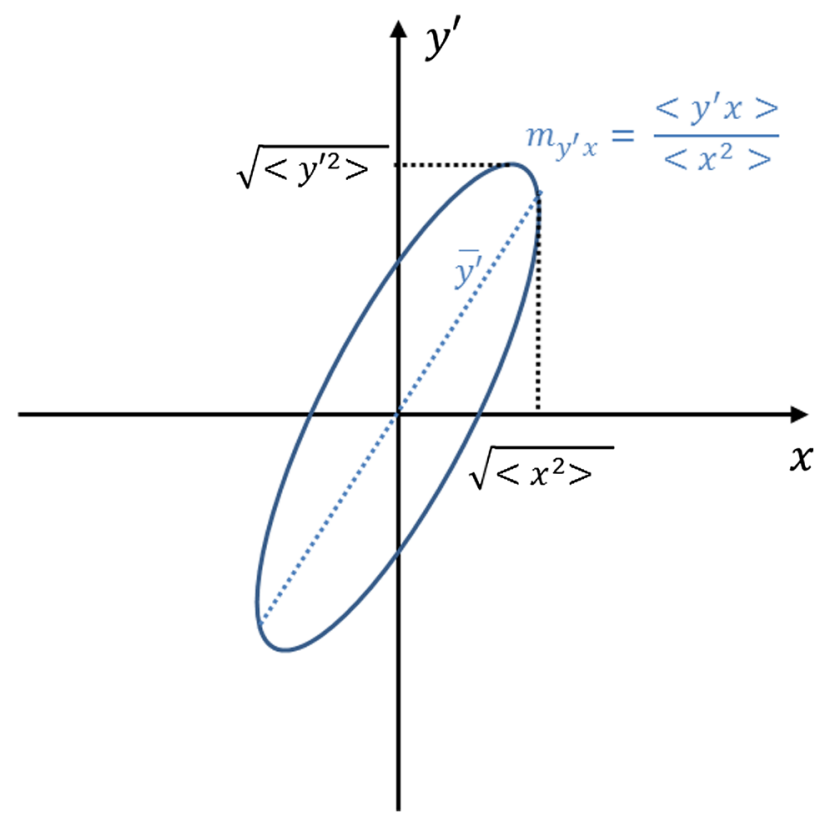

FIG. 3. Projection of the four-dimensional rms-ellipsoid onto the $\left(x, y^{\prime}\right)$ plane and the constant slope $\left(\partial \overline{y^{\prime}} / \partial x\right)$.

where $\overrightarrow{r^{\prime}}(x, y, s):=\left[\overline{x^{\prime}}(x, y, s), \overline{y^{\prime}}(x, y, s), 1\right]$. This ansatz is supported by the similarity of $\mathcal{W}_{A}$ to the first term of Eq. (4). In continuum mechanics, the rotation of a media's velocity $(\vec{\nabla} \times \vec{v})$ is called the vorticity.

As $\mathcal{W}_{A}$ by construction is invariant under rotation by any angle in the $(x, y)$ plane, Eq. (16) may be worked out for a beam with $\langle x y\rangle=0$ without loss of generality (imagine that prior to the calculation of $\mathcal{W}_{A}$ the beam is rotated around the beam axis by an angle that puts $\langle x y\rangle$ to zero). For the following procedure, the resulting beam rms-area (divided by $\pi$ ) $A=\sqrt{\left\langle x^{2}\right\rangle\left\langle y^{2}\right\rangle}$ is treated as being infinitesimally small in the paraxial approximation. Accordingly, the transverse components of $\overrightarrow{\vec{r}}$ are expressed through the first terms of the Taylor series

$$
\begin{aligned}
& \overline{x^{\prime}(x, y)}:=\overline{x^{\prime}}(0,0)+\frac{\partial \overline{x^{\prime}}}{\partial x} \cdot x+\frac{\partial \overline{x^{\prime}}}{\partial y} \cdot y, \\
& \overline{y^{\prime}}(x, y):=\overline{y^{\prime}}(0,0)+\frac{\partial \overline{y^{\prime}}}{\partial x} \cdot x+\frac{\partial \overline{y^{\prime}}}{\partial y} \cdot y,
\end{aligned}
$$

which turns into

$$
\begin{aligned}
& \overline{x^{\prime}}(x, y):=\frac{\left\langle x^{\prime} x\right\rangle}{\left\langle x^{2}\right\rangle} x+\frac{\left\langle x^{\prime} y\right\rangle}{\left\langle y^{2}\right\rangle} y, \\
& \overline{y^{\prime}}(x, y):=\frac{\left\langle y^{\prime} x\right\rangle}{\left\langle x^{2}\right\rangle} x+\frac{\left\langle y^{\prime} y\right\rangle}{\left\langle y^{2}\right\rangle} y .
\end{aligned}
$$

Figure 3 illustrates as an example the constant slope $\left(\partial \overline{y^{\prime}} / \partial x\right)$ of $\overline{y^{\prime}}$ in the projection of the four-dimensional 


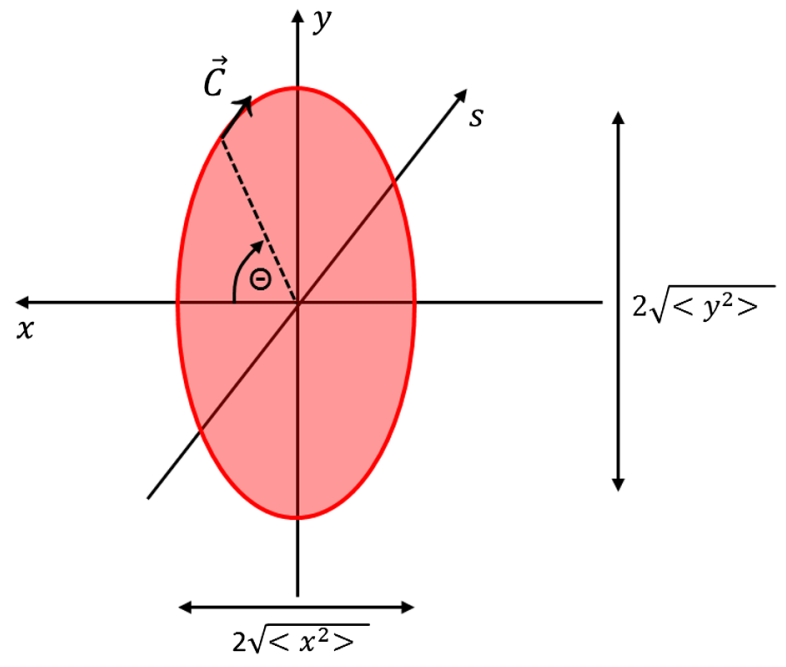

FIG. 4. Visualization of the rms-vorticity $\mathcal{W}_{A}$ for a beam with $\langle x y\rangle=0$. The integration path encloses the elliptical beam rms-area.

rms-ellipsoid onto the $\left(x, y^{\prime}\right)$ plane. The path integral around the rms ellipse $x^{2} /\left\langle x^{2}\right\rangle+y^{2} /\left\langle y^{2}\right\rangle=1$ can be done by the following changes of variables: $x=\sqrt{\left\langle x^{2}\right\rangle} \cos \theta$, $y=\sqrt{\left\langle y^{2}\right\rangle} \sin \theta$, and

$d \vec{C}=\left(\frac{d x}{d \theta}, \frac{d y}{d \theta}\right) d \theta=\left(-\sqrt{\left\langle x^{2}\right\rangle} \sin \theta, \sqrt{\left\langle y^{2}\right\rangle} \cos \theta\right) d \theta$,

as illustrated in Fig. 4. Therefore,

$$
\begin{aligned}
2 A \oint_{\mathcal{C}} \overrightarrow{\vec{r}^{\prime}}(x, y, s) \cdot d \vec{C} \\
=2 A \int_{0}^{2 \pi}\left(\frac{\left\langle x^{\prime} x\right\rangle}{\left\langle x^{2}\right\rangle} x+\frac{\left\langle x^{\prime} y\right\rangle}{\left\langle y^{2}\right\rangle} y\right)\left(-\sqrt{\left\langle x^{2}\right\rangle} \sin \theta\right) d \theta \\
\quad+2 A \int_{0}^{2 \pi}\left(\frac{\left\langle y^{\prime} x\right\rangle}{\left\langle x^{2}\right\rangle} x+\frac{\left\langle y^{\prime} y\right\rangle}{\left\langle y^{2}\right\rangle} y\right)\left(\sqrt{\left\langle y^{2}\right\rangle} \cos \theta\right) d \theta \\
=\left\langle y^{\prime} x\right\rangle\left\langle y^{2}\right\rangle-\left\langle x^{\prime} y\right\rangle\left\langle x^{2}\right\rangle=\mathcal{W}_{A},
\end{aligned}
$$

which proves that the ansatz is correct.

For the time being, acceleration has not been included into the treatment. This can be done simply by multiplying Eqs. (1) and (2) initially by $p=m \gamma \beta c$, where $\beta$ is the longitudinal particle velocity normalized to the velocity of light $c$. The extension of Busch's theorem to beams including acceleration is

$\left(\varepsilon_{n 1}-\varepsilon_{n 2}\right)^{2}+\left[\frac{e q \psi}{m c \pi}\right]^{2}+\frac{4 e q \psi \beta \gamma}{m c \pi} \oint_{\mathcal{C}} \overrightarrow{\vec{r}^{\prime}} \cdot d \vec{C}=$ const,

where $\psi$ is the magnetic flux through the beam rms-area $A$. Analogue to the normalized emittance $\varepsilon_{n}:=\beta \gamma \varepsilon$, the normalized beam rms-vorticity is introduced as

$$
\mathcal{W}_{A n}:=\beta \gamma \mathcal{W}_{A}
$$

One may interpret the squared difference of eigenemittances as intrinsic anisotropy of the beam. Once correlations were removed, intrinsic anisotropy manifests through different beam temperatures (emittances) along two perpendicular degrees of freedom. Using this interpretation Busch's theorem extended to accelerated beams without cylindrical symmetry essentially states

$$
\begin{aligned}
& \text { intrinsic anisotropy }+(\text { magnetic flux })^{2} \\
& \quad+\text { vorticity around magnetic field } \\
& =\text { constant. }
\end{aligned}
$$

\section{TRACKING AND COURANT-SNYDER FORMALISM}

Tracking simulations applying magnetic field maps with finite fringe fields using the BEAMPATH code [18] have been performed in order to verify Eq. (23). The probe beam line (Fig. 5) comprises a solenoid with an extended fringe field, a skewed quadrupole magnet quartet, and another extended solenoid. Figure 5 plots the beam widths, rms-area, the

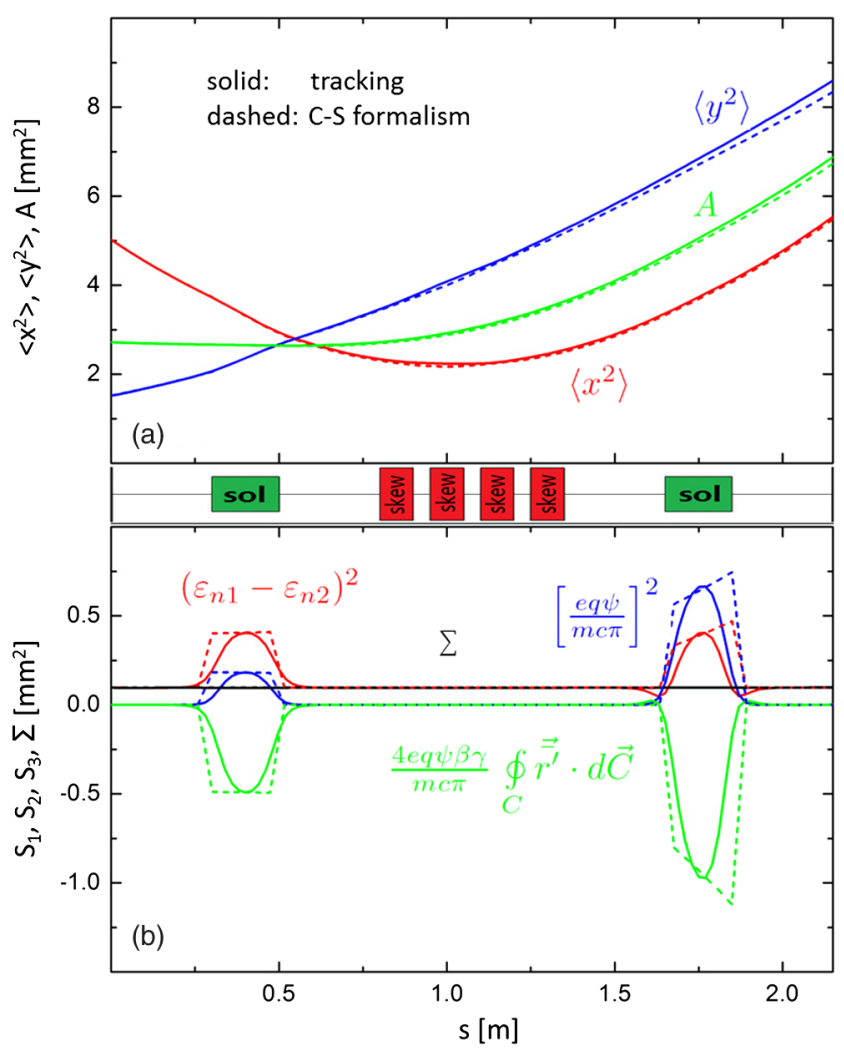

FIG. 5. (a) Horizontal/vertical beam size (red/blue) and beam rms-area (green) along the beam line. (b) Summands of Eq. (23) and their sum along the beam line. Results from tracking (application of the generalized Courant-Snyder formalism) are plotted in solid (dashed). 
three summands of Eq. (23), and their sum along the beam line. Additionally, the results from the application of the generalized Courant-Snyder (C-S) formalism for coupled lattices $[19,20]$ are plotted. In the latter, hard edge fields with infinite short fringe field lengths have been assumed. Slight differences between the envelopes are due to the fact that the $\mathrm{C}-\mathrm{S}$ formalism used hard edge magnetic fields, while tracking was done through magnetic field maps with extended fringe fields. In both cases the three summands change exclusively along regions with a longitudinal magnetic field. Behind these regions, each of them gets back to the value it had prior to entering this region, respectively. The sum of the three beam properties remains constant in accordance to Eq. (23) for the analytical calculations as well as for the tracking simulations. In particular, it is emphasized that the extended theorem holds along the skewed quadrupole magnet region as well, where cylindrical symmetry is absent.

\section{MODELING OF EXPERIMENTS ON EMITTANCE REPARTITIONING}

The extended theorem includes acceleration but it does not require laminar flow. Accordingly, it is applicable to a manifold of scenarios that do advanced beam emittance manipulations such as relativistic electron cooling for instance [21]. In this section the extended theorem is used to model recent experiments of emittance repartitioning through changing the set of eigenemittances in laboratory coordinates. The first one was performed with electrons to form flat beams that may be used in linear colliders to enhance the collision luminosity. The second experiment produced flat ion beams that are injected into subsequent ring accelerators with significantly enlarged efficiency compared to round beams $[16,17]$.

\section{A. Flat electron beam creation}

At FERMILAB's NICADD photoinjector, flat electron beams were formed by first producing the beams at the surface of a photo cathode placed inside an rf-gun to which longitudinal magnetic field $B_{s}=B_{0}$ was imposed [11]. The schematic beam line is sketched in Fig. 6.

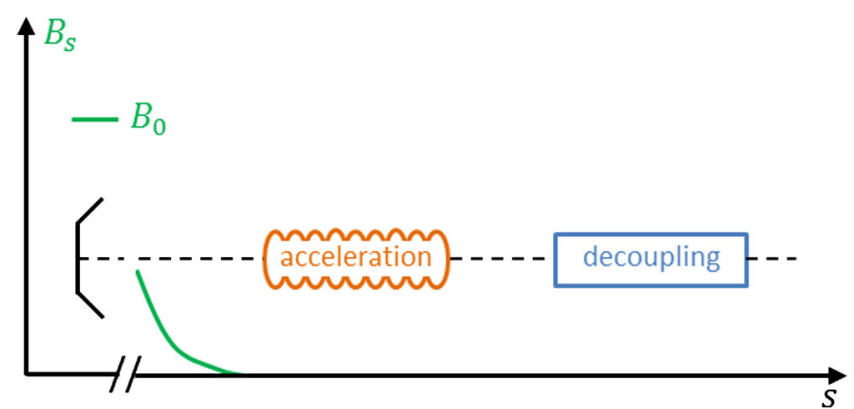

FIG. 6. Schematic sketch of the beam line of the experiment performed at NICCAD at FERMILAB [11].
Along the subsequent region with $B_{s}=0$, the beam was accelerated to $16 \mathrm{MeV}$. Finally, correlations initially imposed by the magnetic exit fringe field of the rf-gun were removed by three skew quadrupole magnets. Equation (23) equalizes the situation at the cathode surface at the left-hand side to the situation of the finally flat beam on the right-hand side $(q=1)$

$$
0+\left[\frac{e B_{0} A_{0}}{m c}\right]^{2}+0=\left(\varepsilon_{n f 1}-\varepsilon_{n f 2}\right)^{2}+0+0,
$$

where $A_{0}$ is the beam rms-area at the cathode surface. The authors of [11] used the definitions [22]

$$
\begin{gathered}
\left(\varepsilon_{n}^{u}\right)^{2}:=\varepsilon_{n f 1} \cdot \varepsilon_{n f 2} \\
\mathcal{L}:=\left(e B_{0} A_{0}\right) /(2 m \gamma \beta c)
\end{gathered}
$$

resulting in

$$
\varepsilon_{n f 1}=\mathcal{L} \beta \gamma \pm \sqrt{(\mathcal{L} \beta \gamma)^{2}+\left(\varepsilon_{n}^{u}\right)^{2}},
$$

of which only the upper sign gives a meaningful positive result. Replugging this expression for $\varepsilon_{n f 1}$ into Eq. (25) leads to

$$
\varepsilon_{n f 1 / 2}= \pm \mathcal{L} \beta \gamma+\sqrt{(\mathcal{L} \beta \gamma)^{2}+\left(\varepsilon_{n}^{u}\right)^{2}},
$$

being identical to their original expression (Eq. (1) of [11]).

\section{B. Emittance transfer experiment EMTEX}

At GSI, the EMittance Transfer EXperiment (EMTEX) transferred emittance from one transverse plane into the other one by passing the beam through a short solenoid [8,15-17]. The schematic EMTEX beam line is depicted in Fig. 7. In the solenoid center, the ions charge state, i.e., their rigidity was changed by placing a thin carbon foil therein

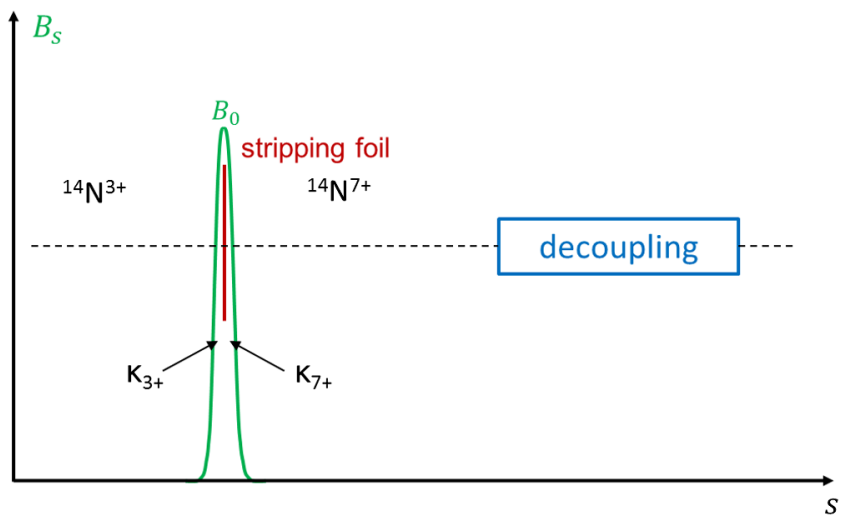

FIG. 7. Schematic beam line of EMTEX at GSI for transverse emittance transfer [16]. 
TABLE I. Beam parameters of EMTEX [16].

\begin{tabular}{lc}
\hline \hline Parameter & Value \\
\hline Kinetic energy & $11.45 \mathrm{MeV} / \mathrm{u}$ \\
Mass number & 14 \\
$\varepsilon_{x, 3+}$ & $1.040 \mathrm{~mm} \mathrm{mrad}$ \\
$\varepsilon_{y, 3+}$ & $0.825 \mathrm{~mm} \mathrm{mrad}$ \\
$\beta_{x, 3+}$ & $3.461 \mathrm{~m}$ \\
$\beta_{y, 3+}$ & $5.845 \mathrm{~m}$ \\
$q_{\text {in/out }}$ & $3+/ 7+$ \\
$(B \rho)_{3+/ 7+}$ & $2.278 / 0.976 \mathrm{Tm}$ \\
$B_{0}$ & $0.9 \mathrm{~T}$ \\
\hline \hline
\end{tabular}

from ${ }^{14} \mathrm{~N}^{3+}$ to ${ }^{14} \mathrm{~N}^{7+}$. Charge state stripping is a standard procedure used at several laboratories that deliver heavy or intermediate mass ions $[23,24]$. In front of the solenoid, the beam had no interplane correlations, and thus, the difference of rms-emittances was equal to the difference of eigenemittances (mod. sign). Since the solenoid was short, the beam area at the foil $A:=A_{f}$ can be approximated as constant during the beam transit through the solenoid. Table I lists the relevant parameters of the experiment. Equation (13) relates the beam parameters in front of the solenoid $\left(B_{s}=0\right.$, no correlations $\rightarrow \mathcal{W}_{A}=0, \varepsilon_{10}=\varepsilon_{x, 3+}$, $\varepsilon_{20}=\varepsilon_{y, 3+}$ ) to those in front of the foil in the center of the short solenoid:

$$
\begin{aligned}
& \left(\varepsilon_{x, 3+}-\varepsilon_{y, 3+}\right)^{2}+0+0 \\
& =\left(\varepsilon_{1 f}-\varepsilon_{2 f}\right)^{2}+\left[\frac{A_{f} B_{0}}{(B \rho)_{3+}}\right]^{2}+\frac{2 B_{0}}{(B \rho)_{3+}} \mathcal{W}_{A f},
\end{aligned}
$$

where the index $f$ refers to the location of the foil. The entrance fringe field of the solenoid causes the rmsvorticity

$$
\mathcal{W}_{A_{f}}=\Delta \mathcal{W}_{A}=-2 \kappa_{3+} \cdot A_{f}^{2}=-2 \frac{B_{0}}{2(B \rho)_{3+}} A_{f}^{2}
$$

leading to

$\left(\varepsilon_{x, 3+}-\varepsilon_{y, 3+}\right)^{2}+0+0=\left(\varepsilon_{1 f}-\varepsilon_{2 f}\right)^{2}-\left[\frac{A_{f} B_{0}}{(B \rho)_{3+}}\right]^{2}$.

Using the initial beam parameters of the experiment from Table I, $A_{f}=\sqrt{\varepsilon_{x} \beta_{x} \varepsilon_{y} \beta_{y}}=4.166 \mathrm{~mm}^{2}$, and the identity $1 \mathrm{mmmrad}=1 \mu \mathrm{m}$ gives

$$
\begin{aligned}
\left(\varepsilon_{1 f}-\varepsilon_{2 f}\right)^{2} & =\left(\varepsilon_{x, 3+}-\varepsilon_{y, 3+}\right)^{2}+2.709 \mu \mathrm{m}^{2} \\
& =2.755 \mu \mathrm{m}^{2} .
\end{aligned}
$$

Equation (13) is re-used to relate the beam parameters just behind the foil but still at the center of the solenoid to those at the exit of the beam line, where $B_{s}=0$ and the beam correlations have been removed again. Angular scattering in the foil is neglected. As the beam changed rigidity in the foil, $(B \rho)_{3+}$ must be properly replaced by $(B \rho)_{7+}$. However, second beam moments are not changed by the foil, i.e., $\mathcal{W}_{A}=\mathcal{W}_{A_{f}}$, right in front and right behind the foil. Accordingly,

$$
\begin{aligned}
& \left(\varepsilon_{1 f}-\varepsilon_{2 f}\right)^{2}+\left[\frac{A_{f} B_{0}}{(B \rho)_{7+}}\right]^{2}+\frac{2 B_{0}}{(B \rho)_{7+}} \mathcal{W}_{A f} \\
& =\left(\varepsilon_{x, 7+}-\varepsilon_{y, 7+}\right)^{2}+0+0,
\end{aligned}
$$

which by using Eq. (31) and plugging in the values delivers

$$
\left|\varepsilon_{x, 7+}-\varepsilon_{y, 7+}\right|=2.2 \mathrm{~mm} \mathrm{mrad}
$$

fitting well the measured value of $2.0 \mathrm{~mm}$ mrad (see Fig. 2 of [16]).

\section{SUMMARY}

The many particle pendant to Busch's theorem on a single particle has been derived without requiring cylindrical symmetry but with including acceleration of the beam. It introduces the property of beam rms-vorticity and relates the beam's difference of eigenemittances (i.e., intrinsic anisotropy), the magnetic flux through its area, and its rms-vorticity multiplied by the magnetic flux. Under the transport through coupled linear elements, the sum of these properties is preserved. The extended theorem was verified through tracking simulations with field maps and through application of the generalized $\mathrm{C}-\mathrm{S}$ formalism for coupled dynamics. It was successfully used for quick and precise modeling of emittance re-partitioning experiments conducted at FERMILAB and at GSI, hence it is a powerful tool easily applicable to both electron and ion beam lines or accelerators. Prior to its formulation scenarios for advanced beam manipulations based on interplane coupling were developed quite separately for ion or electron applications. Busch's original theorem for instance was hardly applied to ion beams. The extended theorem instead forms a common ground for both applications providing a strong connection between them. It significantly facilitates modeling and designing of devices for emittance manipulations.

\section{ACKNOWLEDGMENTS}

This research was partly supported by the National Research Foundation of Korea (Grants No. NRF-2015R1D1A1A01061074 and No. NRF2017M1A7A1A02016413).

[1] H. Busch, Berechnung der Bahn von Kathodenstrahlen in axial symmetrischen electromagnetischen Felde, Ann. Phys. (Berlin) 386, 974 (1926). 
[2] M. Reiser, Theory and Design of Charged Particle Beams, 2nd ed. (Wiley-VCH, Weinheim, 2008), Chap. 2.

[3] S. E. Tsimring, Electron Beams and Microwave Vacuum Electronics (John Wiley \& Sons, New York, 2007), Chap. 1 and 3.

[4] P. T. Kirstein, G. S. Kino, and W. E. Waters, Space Charge Flow (McGraw-Hill, New York, 1967), p. 14.

[5] A. J. Dragt, General moment invariants for linear Hamiltonian systems, Phys. Rev. A 45, 2572 (1992).

[6] K. Floettmann, Some basic features of the beam emittance, Phys. Rev. ST Accel. Beams 6, 034202 (2003).

[7] Emittance definitions assume mono-energetic beams and refer to fixed position $s_{0}$ rather to fixed time $t_{0}$. The particle angle $u^{\prime}$ and its transverse mechanical momentum $P_{u}:=$ $m \gamma v_{u}$ are related through $P_{u}=p \cdot u^{\prime}$, where $p$ is the total mechanical momentum, which is the same for each particle.

[8] C. Xiao, L. Groening, O. Kester, H. Leibrock, M. Maier, and C. Mühle, Single-knob beam line for transverse emittance partitioning, Phys. Rev. ST Accel. Beams 16, 044201 (2013).

[9] R. Brinkmann, Y. Derbenev, and K. Flöttmann, Report No. DESY TESLA-99-09, 1999.

[10] R. Brinkmann, Y. Derbenev, and K. Flöttmann, A low emittance, flat-beam electron source for linear colliders, Phys. Rev. ST Accel. Beams 4, 053501 (2001).

[11] P. Piot, Y.-E Sun, and K.-J. Kim, Photoinjector generation of a flat electron beam with transverse emittance ratio of 100, Phys. Rev. ST Accel. Beams 9, 031001 (2006).

[12] A. Burov, S. Nagaitsev, and Y. Derbenev, Circular modes, beam adapters, and their applications in beam optics, Phys. Rev. E 66, 016503 (2002).

[13] A. Burov, Circular modes for flat beams in the LHC, Phys. Rev. ST Accel. Beams 16, 061002 (2013).

[14] P. Bertrand, J. P. Biarrotte, and D. Uriot, Flat beams and application to the mass separation of radioactive beam, in Proceedings of the 10th European Particle Accelerator Conference, Edinburgh, Scotland, edited by J. Poole and C. Petit-Jean-Genaz (Institute of Physics, Edinburgh, Scotland, 2006).

[15] L. Groening, Concept for controlled transverse emittance transfer within a linac ion beam, Phys. Rev. ST Accel. Beams 14, 064201 (2011).
[16] L. Groening, M. Maier, C. Xiao, L. Dahl, P. Gerhard, O. K. Kester, S. Mickat, H. Vormann, and M. Vossberg, Experimental Proof of Adjustable Single-Knob Ion Beam Emittance Partitioning, Phys. Rev. Lett. 113, 264802 (2014).

[17] L. Groening, S. Appel, L. Bozyk, Y. El-Hayek, M. Maier, and C. Xiao, Demonstration of flat ion beam-creation and injection into a synchrotron, in Proceedings of the 6th International Particle Accelerator Conference, Richmond, VA, USA, edited by S. Henderson (ANL, Richmond, 2015).

[18] Y. K. Batygin, Particle-in-cell code BEAMPATH for beam dynamics simulations in linear accelerators and beamlines, Nucl. Instrum. Methods Phys. Res., Sect. A 539, 455 (2005).

[19] H. Qin, R. C. Davidson, J. W. Burby, and M. Chung, Analytical methods for describing charged particle dynamics in general focusing lattices using generalized CourantSnyder theory, Phys. Rev. ST Accel. Beams 17, 044001 (2014).

[20] M. Chung, H. Qin, R. C. Davidson, L. Groening, and C. Xiao, Generalized Kapchinskij-Vladimirskij Distribution and Beam Matrix for Phase-Space Manipulations of High-Intensity Beams, Phys. Rev. Lett. 117, 224801 (2016).

[21] A. Burov, S. Nagaitsev, A. Shemyakin, and Ya. Derbenev, Optical principles of beam transport for relativistic electron cooling, Phys. Rev. ST Accel. Beams 3, 094002 (2000).

[22] K.-J. Kim, Round-to-flat transformation of angularmomentum-dominated beams, Phys. Rev. ST Accel. Beams 6, 104002 (2003).

[23] H. Okuno, N. Fukunishi, A. Goto, H. Hasabe, H. Imao, O. Kamigaito, M. Kase, H. Kuboki, Y. Yano, and S. Yokouchi, Low-Z gas stripper as an alternative to carbon foils for the acceleration of high-power uranium beams, Phys. Rev. ST Accel. Beams 14, 033503 (2011).

[24] P. Scharrer, Ch. E. Düllmann, W. Barth, J. Khuyagbaatar, A. Yakushev, M. Bevcic, P. Gerhard, L. Groening, K. P. Horn, E. Jäger, J. Krier, and H. Vormann, Measurements of charge state distributions of 0.74 and $1.4 \mathrm{MeV} / \mathrm{u}$ heavy ions passing through dilute gases, Phys. Rev. Accel. Beams 20, 043503 (2017). 